\title{
Überforderte pflegende Angehörige bei der Versorgung am Lebensende - was bieten Hausarztpraxen zur Entlastung an?
}

\section{Burden Experienced by Family Caregivers of Patients at the End of Life: What do General Practice Teams Offer?}

Autoren

Institut
K. Krug, R. Bölter, R. A. Ballhausen, P. Engeser, F. Peters-Klimm

Abteilung Allgemeinmedizin und Versorgungsforschung, Universitätsklinikum Heidelberg, Heidelberg
Schlüsselwörter

- pflegende Angehörige

- hausärztliche Praxisteams

- Unterstützung

- Palliativversorgung

- Netzwerk

Key words

- family caregivers

- general practice teams

- social support

- palliative care

- network
Bibliografie

DOI http://dx.doi.org/

10.1055/s-0042-111206

Online-Publikation: 21.7.2016

Gesundheitswesen 2016;

78 (Suppl. 1): e128-e134

(c) Georg Thieme Verlag KG

Stuttgart · New York

ISSN 0949-7013

Korrespondenzadresse

Dr. sc. hum. Katja Krug,

Abteilung Allgemeinmedizin und Versorgungsforschung Universitätsklinikum Heidelberg Im Neuenheimer Feld 130.3 69120 Heidelberg

katja.krug@med.uni-

heidelberg.de

\section{License terms}

\section{(ब(1) $\Theta \circledast$}

\section{Zusammenfassung}

$\nabla$

Ziel der Studie: war zu eruieren, inwiefern hausärztliche Praxisteams darauf vorbereitet sind, pflegende Angehörige von Palliativpatienten zu entlasten, welche Unterstützung sie konkret anbieten und wo sie Verbesserungsbedarf sehen.

Methodik: Mit Praxisteams (Hausärzte, HÄ, und Medizinische Fachangestellte, MFA) wurden Fokusgruppen und Interviews zu den Themen Identifikation und Unterstützung pflegender Angehöriger durchgeführt und qualitativ ausgewertet.

Ergebnisse: Die 21 Teilnehmer (14 HÄ, 7 MFA) aus 13 Praxen erkannten überforderte Angehörige, boten daraufhin Unterstützung und vermittelten Kontakte zu lokalen Beratungsangeboten. Sie wiesen Angehörige auf ihr soziales Netzwerk hin, damit sie sich Freiräume für eigene Bedürfnisse schaffen können.

Schlussfolgerung: Praxisteams weisen eine Vielzahl individualisierter und unsystematischer Herangehensweisen zur Unterstützung von pflegenden Angehörigen auf. Folgeuntersuchungen im Rahmen des Projekts sollen systematische Ansätze identifizieren und erproben. Gefördert vom Bundesministerium für Bildung und Forschung (BMBF), Förderkennzeichen: 01GX1046

\section{Einleitung \\ $\nabla$}

Ein Großteil der Versorgung am Lebensende wird ambulant vorgenommen, was den Patienten eine individuelle Lebensgestaltung erlaubt und ihre Lebensqualität verbessert [1,2]. Einflussfaktoren auf den Ort der Betreuung am Lebensende sind vor allem die soziale Unterstützung durch Laien, die Präferenzen der Patienten und die Kontakte zu Versorgern im Gesundheitswesen [3].

\section{Abstract}

$\nabla$

The aim of the study: was to determine how far general practice teams are prepared to relieve family caregivers of palliative patients from their caregiving burden, the support they actually offer, and where they identify needs for improvement.

Method: Focus groups and interviews on the issues of identification and support of family caregivers were conducted with practice teams (general practitioners, GPs, and medical assistants, MAs) and the results qualitatively analyzed.

Results: 21 participants (14 GPs, 7 MAs) from 13 practices identified burdened family caregivers, thereupon offered support and provided contact details to local consultation services. They suggested to family caregivers that they should use their social network to create room for meeting their own needs.

Conclusions: Practice teams use a multitude of individualized and unsystematic approaches to support family caregivers. In further studies within the framework of this project, systematic approaches will be identified and tried out.
Zu den Laien gehören Familienangehörige, Freunde und Nachbarn. Diese pflegenden Angehörigen sind - im Gegensatz zu den (meisten) professionellen Versorgern - oftmals zeitlich umfangreich für die Patienten da [4]. Um genügend Unterstützung bei der Versorgung und Pflege der Patienten zu leisten, wünschen sich pflegende Angehörige Wissen über die Symptome der Palliativpatienten, Zugang zu Informationen [5] sowie spirituelle Unterstützung [6]. Pflegende Angehörige sind nicht nur körperlich gefordert, sie entwickeln 
häufig auch psychische Symptome [7] und benötigen selbst Unterstützung.

In Deutschland rücken pflegende Angehörige seit den 1990er Jahren verstärkt in den Fokus der Forschung [8], nicht zuletzt auch im Zuge der bundesweiten Einführung der Pflegeversicherung und der Einrichtung von Pflegestützpunkten. So gibt es lokale Projekte z.B. zur Unterstützung pflegender Kinder und Jugendlicher [9]. Ebenfalls wurden Herausforderungen pflegender Angehöriger unterschiedlicher Gruppen, z.B. von Immigranten [10] und pflegenden Angehörigen von Demenzerkrankten untersucht (z.B. LEANDER-Studie [11], IDA-Projekt [12], Entlastungsprogramm bei Demenz - EDe [13]). Die Ergebnisse der Demenz-Projekte lassen sich nicht ohne Probleme auf pflegende Angehörige von Patienten mit anderen Grunderkrankungen übertragen. So waren Interventionseffekte bei Angehörigen von Demenzerkrankten kleiner als bei anderen pflegenden Angehörigen [14]. Auch die Pflegekassen nehmen pflegende Angehörige als wichtige Ressource in der Versorgung wahr, die unterstützt werden muss, z. B. durch Pflegekurse. Außerdem gibt die Unfallkasse NRW regelmäßig die Zeitschrift „Angehörige pflegen“ (http://www.unfallkasse-nrw.de/gesundheitsdienstportal/ index.html) heraus.

Seit April 2007 wurden Angebote der Spezialisierten Ambulanten Palliativversorgung (SAPV) gesetzlich verankert ( $\S 37 \mathrm{~b}$ Sozialgesetzbuch V), die für etwa $10 \%$ der Palliativpatienten mit komplexem Symptomgeschehen benötigt werden. Aussagen zur allgemeinen ambulanten Palliativversorgung, die etwa 90\% der Patienten betrifft, fehlen. In der ambulanten Versorgung sollen laut dem Grundsatz „ambulant vor stationär“ [15] unnötige Einweisungen vermieden werden. Patienten, bei denen Angehörige in die Versorgung einbezogen sind, werden am Lebensende seltener stationär eingewiesen und versterben häufiger im häuslichen Umfeld [16].Wenn es in den letzten Lebenstagen zu Krankenhauseinweisungen kommt $[17,18]$, dann auch deshalb, weil sich pflegende Angehörige mit der Situation überfordert fühlen und nicht ausreichend bedarfsgerechte professionelle Unterstützung erhalten [19].

Hausärzte (HÄ) und ihre Praxisteams (Medizinische Fachangestellte, MFA) gehören zu den ersten Ansprechpartnern für Patienten und Angehörige im Gesundheitssystem, sie übernehmen den Großteil der ambulantenprimärärztliche Versorgung und kennen Patienten und Angehörige oft über einen längeren Zeitraum[2]. Damit kommt ihnen auch die Aufgabe zu, Belastungen von pflegenden Angehörigen rechtzeitig zu erkennen und Entlastungsmöglichkeiten aufzuzeigen. Für Hausärzte erschien in Deutschland 2005 die Leitlinie „Pflegende Angehörige“ der Deutschen Gesellschaft für Allgemeinmedizin und Familienmedizin (DEGAM) [20]. Die Autoren haben ein allgemein gültiges Informationsblatt für Angehörige beigelegt, weisen in diesem Zusammenhang aber darauf hin, dass „Informationen über regionale Angebote [...] spezifisch für den Praxisbereich vom Hausarzt-Team selbst ausgearbeitet und zur Verfügung gestellt werden" sollten ([20], S. 23). Studien, die eine Umsetzung der Leitlinie in die Praxis untersuchen, fehlen bisher.

Im Rahmen eines Pilotprojekts zur Unterstützung pflegender Angehöriger von Palliativpatienten in der Hausarztpraxis (PalliPA) [21]wird in dieser Arbeit zunächst die aktuelle Situation in Hausarztpraxen untersucht:(1) welche Unterstützung bieten sie konkret zur Entlastung pflegender Angehörige an und (2) wo sehen sie Verbesserungsbedarf.

\section{Methode}

$\nabla$

\section{Studiendesign}

Die Fragestellungen wurden mit einem qualitativen Studiendesign beantwortet. Dazu wurden Fokusgruppen mit hausärztlichen Praxisteams durchgeführt.

\section{Stichprobe und Rekrutierung}

Das Projekt zielte darauf ab, gemeinsam mit hausärztlichen Praxisteams Strategien zur Entlastung pflegender Angehöriger von Palliativpatienten in den Praxen zu entwickeln und zu implementieren [21]. Um Praxisteams zu gewinnen, die ein großes Interesse am Thema hatten, wurden nur Praxen zur Teilnahme eingeladen, in denen mindestens ein Arzt/eine Ärztin die Zusatzbezeichnung Palliativmedizin führte. Das Projekt war auf Baden-Württemberg beschränkt. Zum Zeitpunkt der Versendung der Einladungen (März 2011) waren bei der Kassenärztlichen Vereinigung Baden-Württemberg 258 geeignete Praxen geführt, die alle zur Teilnahme am Projekt eingeladen wurden.

\section{Leitfadenentwicklung}

Der Leitfaden zur Moderation der Fokusgruppen wurde von allen Autoren gemeinsam erstellt und beinhaltete 4 Schlüsselthemen: (1) Komponenten einer optimalen Palliativversorgung, (2) Identifikation belasteter pflegender Angehöriger in der Praxis, (3) Unterstützung der Angehörigen durch das Praxisteam und (4) Verbesserungswünsche.

\section{Datenerhebung und Auswertung}

Die Fokusgruppen fanden im Anschluss an Informationsveranstaltungen zur Studie statt. Dabei erhielten die Teilnehmer eine Übersicht über die geplante Studie, deren theoretische Hintergründe wurden vorgestellt und nationale (z.B. die DEGAMLeitlinie Pflegende Angehörige) und internationale (z.B. Gold Standards Framework) Ansätze zur Entlastung pflegender Angehöriger genannt, ohne näher auf Einzelheiten einzugehen. Die Fokusgruppen und die Interviews wurden protokolliert, aufgezeichnet, transkribiert und inhaltsanalytisch nach Mayring [22] ausgewertet. Dazu wurde das Material zunächst gesichtet, aus den Leitfragen deduktive Kategorien abgeleitet und von 2 Wissenschaftlern (KK, RAB) unabhängig mithilfe von ATLAS.ti kodiert. Induktive Kategorien wurden mit einem weiteren Wissenschaftler (FPK) konsentiert.

Soziodemografische Daten wie Alter, Berufserfahrung oder Praxistätigkeit wurden nicht separat erhoben, sind von den Teilnehmern im Rahmen der Vorstellung in der Fokusgruppe aber teilweise genannt worden.

\section{Ethische Aspekte}

Für das Projekt liegt ein positives Votum der Ethikkommission der Universität Heidelberg vor (S-042/2011).

\section{Ergebnisse \\ $\nabla$}

Von den 258 eingeladenen antworteten 35 Praxen (14\%), $22 \mathrm{mit}$ einer sofortigen Absage. 11 Praxen (12 HÄ, 7 MFA) nahmen an den Informationsveranstaltungen und einer der insgesamt 4 90-minütigen Fokusgruppen teil, die von KK, PE und FPK anhand des Leitfadens moderiert wurden. Mit 2 HÄ führte KK zusätzlich Telefoninterviews durch, da sie an dem Projekt teilnehmen wollten, aber wegen Terminschwierigkeiten nicht zu den Informa- 
tionsveranstaltungen kommen konnten. Charakteristika der Teilnehmer sind in $\bullet$ Tab. 1 aufgeführt.

\section{Unterstützung für pflegende Angehörige durch \\ Praxisteams}

Sowohl Hausärzte als auch MFA äußerten sich zur Unterstützung für pflegende Angehörige. Verschiedene Elemente der Unterstützung wurden dabei angesprochen: (1) Sie waren der Meinung, dass sie sowohl die Bedürfnisse der Angehörigen einschätzen konnten als auch erkannten, wenn die Angehörigen mit der Situation überfordert waren. (2) Sie gaben an, dass sie über den anstehenden Krankheitsprozess aufklärten, psychosoziale und bürokratische Unterstützung anboten und Kontakte zu lokalen Beratungs- und Betreuungsangeboten vermittelten. (3)
Des Weiteren äußerten sie sich dazu, dass sie die Angehörigen ermutigten, ihr soziales Netzwerk zu nutzen, um Freiräume für ihre eigenen Bedürfnisse zu schaffen.

\section{(1) Einschätzung der Bedürfnisse und Überforderung der Angehörigen}

Die Praxisteams gaben an, dass die Bedürfnisse der Angehörigen entweder von den HÄ oder den Angehörigen selbst direkt angesprochen wurden. Die HÄ erklärten, dass Angehörige direkt berichteten, wenn sie überfordert waren. Allerdings erwähnten die HÄ auch, dass Angehörige indirekte Überforderung zeigten, indem sie eigene Symptome ansprachen ( $\bullet$ Tab. 2). Die HÄ berichteten, dass sie die Angehörigen unter 4 Augen auch gezielt nach ihren Belastungen befragten. Die Praxisteams äußerten, dass sie

Tab. 1 Charakteristika der Teilnehmer.

\begin{tabular}{|c|c|c|c|c|}
\hline & Kürzel & Geschlecht & Anmerkungen & berufliche Erfahrung \\
\hline \multirow[t]{5}{*}{ Fokusgruppe 1} & MFA1 & w & $\begin{array}{l}\text { VERAH plus } \\
\text { Praxis von HA3 }\end{array}$ & seit 2 Jahren VERAH \\
\hline & MFA2 & w & Praxis von HA4 & keine Angaben \\
\hline & MFA3 & w & Praxis von HA4 & seit 10 Jahren in der Praxis \\
\hline & HA1 & $\mathrm{m}$ & mit MFA5 & Niederlassung in der Praxis seit 22 Jahren, seit 1 Jahr Palliativmediziner \\
\hline & $\mathrm{HA} 2$ & $\mathrm{~m}$ & mit MFA4 & Niederlassung in der Praxis seit 26 Jahren, seit 5 Jahren Palliativmediziner, SAPV \\
\hline \multirow[t]{5}{*}{ Fokusgruppe 2} & MFA4 & w & $\begin{array}{l}\text { VERAH } \\
\text { Praxis von HA2 }\end{array}$ & seit 9 Jahren in der Praxis \\
\hline & MFA5 & w & Praxis von HA1 & keine Angaben \\
\hline & HA3 & $\mathrm{m}$ & mit MFA1 & Niederlassung in der Praxis seit 24 Jahren, seit 1 Jahr Palliativmediziner \\
\hline & HA4 & w & mit MFA2 und MFA3 & seit 10 Jahren Allgemeinmediziner, Niederlassung in der Praxis seit 6 Jahren \\
\hline & HA5 & m & & Niederlassung in der Praxis seit 27 Jahren \\
\hline \multirow[t]{4}{*}{ Fokusgruppe 3} & HA6 & w & mit MFA7 & Niederlassung in der Praxis seit 13 Jahren, seit 7 Jahren Palliativmediziner \\
\hline & HA7 & w & mit MFA6 & Niederlassung in der Praxis seit 12 Jahren, seit 3 Jahren Palliativmediziner, SAPV \\
\hline & HA8 & w & & $\begin{array}{l}\text { Niederlassung in der Praxis seit } 1 \text { Jahr, davor } 14 \text { Jahre Neurochirurgie und } 4 \text { Jahre } \\
\text { Allgemeinmedizin }\end{array}$ \\
\hline & HA9 & w & gleiche Praxis wie HA11 & seit 2 Jahren Palliativmediziner \\
\hline \multirow[t]{5}{*}{ Fokusgruppe 4} & MFA6 & w & Praxis von HA7 & seit 1 Jahr in der Praxis, vorher Lehre in einer anderen Praxis abgeschlossen \\
\hline & MFA7 & w & Praxis von HA6 & seit 5 Jahren in der Praxis, vorher im Krankenhaus tätig \\
\hline & HA10 & w & & $\begin{array}{l}\text { Niederlassung in der Praxis seit } 10 \text { Jahren, seit } 5 \text { Jahren Palliativmediziner, seit } \\
\text { mind. } 20 \text { Jahren Kontakt zu Palliativpatienten }\end{array}$ \\
\hline & HA11 & $\mathrm{m}$ & gleiche Praxis wie HA9 & seit 3 Jahren Palliativmediziner \\
\hline & HA12 & w & & Niederlassung in der Praxis seit mind. 20 Jahren, seit 1 Jahr Palliativmediziner \\
\hline Telefoninterview 1 & HA13 & $\mathrm{m}$ & & keine Angaben \\
\hline Telefoninterview 2 & HA14 & w & & Niederlassung in der Praxis seit 3 Jahren, vorher Geriatrie \\
\hline
\end{tabular}

Tab. 2 Einschätzung der Bedürfnisse und Überforderung der Angehörigen.

\section{Code}

Angehörige berichten indirekt über Überforderung anhand von Symptomen Hausärzte fragen Angehörige gezielt nach Belastungen

Verhaltensänderung der Angehörigen bei Überforderung

Verhaltensänderung zwischen Patienten und Angehörigen bei Überforderung

Überforderung des Angehörigen durch Rollenvielfalt

Angehörigen berichten ihre Überforderung der Medizinischen Fachangestellten

\section{Beispielzitat}

„Dass sie dann irgendwann sagen, ich kann nicht mehr schlafen, ich bin so schlapp, ich bin kaputt, ich hab keine Freude mehr." (HA2)

„Oder dann aber das Beste ist, wenn sie halt mal da sind, [...] dass man sie irgendwie so mal geschwind nimmt und sagt, wie geht's Ihnen denn?" (HA9)

„Die Aggressivität und es gibt ja auch andere Formen der Äußerung der Angehörigen.“ (HA11)

„Auch Konfliktsituationen, was man dann so mitkriegt, wenn man dann doch mal auf einem Hausbesuch z. B. ist. [...] Wo die vorher immer recht nett und höflich miteinander umgegangen sind, wenn sich da der Ton ändert. Wenn die plötzlich, also der Pflegende aus einer Nichtigkeit heraus, [...] einfach im Ton und in der Art und Weise, wie umgegangen wird, nicht mehr adäquat ist. Und die das teilweise dann hinterher bereuen und auch Schuldgefühle dann entwickeln. [...] Sich aber nicht trauen sich einzugestehen, dass das eine Überforderungssituation ist. "(HA6) „Dann ist ja schon [ein] Angehöriger, der macht die Pflege. Aber man stellt nachher fest, dass er's in seinem tagtäglichen Handling ja gar nicht gebacken kriegt, weil's einfach zu viel drum rum ist. Man kann ja nicht seinen Beruf zur Seite legen. Man kann seine Familie nicht zur Seite legen. " (HA2)

„Der [MFA] erzählen die [Angehörigen] manchmal auch Sachen, die die mir nicht erzählen. Da platzt das ja auch manchmal raus, wie die belastet sind. Und da heulen die sich auch mal wirklich aus. “(HA7) 
Überforderung ebenfalls aus Beobachtungen wahrnahmen, vor allem bei Veränderungen der Stimmungslage und des Verhaltens der Angehörigen. Dabei gaben sie an, dass sich die Verhaltensänderungen bei den Angehörigen nicht nur auf Besuche in der Praxis bezogen, sondern auch auf das Verhältnis zwischen Patienten und Angehörigen. Dabei berichteten die Praxisteams, dass sie einen veränderten Umgang zwischen Patienten und Angehörigen zum Negativen wahrnahmen. Außerdem stellten sie fest, dass pflegende Angehörige ihrer Rollenvielfalt nicht mehr gerecht würden, die Kontaktfrequenz stiege, die Angehörige eine akute Belastungsreaktion zeigten, eigene Symptome berichteten und sich nicht mehr an Absprachen hielten. Die MFA erwähnten, dass sie ihrerseits Überforderung ebenfalls im Einzelkontakt mit den Angehörigen erkannten, in der Praxis an der Rezeption, im Labor, im Untersuchungszimmer oder auch bei Hausbesuchen. Dabei stellten HÄ und MFA fest, dass der Kontakt der MFA zu den Angehörigen anders zu sein schien als der zwischen Angehörigen und HÄ: einige Angehörige redeten offener mit den MFA, andere eher mit den HÄ.

Insgesamt erklärten die Praxisteams, dass sie ihrer Ansicht nach Belastung von pflegenden Angehörigen auf verschiedene Weise erkannten, sie verneinten aber eine systematische Herangehensweise zur Identifikation von Belastung.

\section{(2) Aufklärung, Unterstützung, Kontaktvermittlung} Die HÄ sagten aus, dass sie im Rahmen der medizinischen Versorgung die Angehörigen für den anstehenden Krankheitsprozess sensibilisierten und sie bei Bedarf auch psychosozial unterstützten ( $\bullet$ Tab. 3). Sie gaben an bei der Beschaffung von Hilfsmitteln zu helfen. Darüber hinaus sagten die Praxisteams, sie übernähmen koordinative Leistungen und unterstützten beim Stellen von Anträgen. Lokale Beratungs- und Betreuungsangebote, bei denen die Angehörigen professionelle Hilfe erfuhren, würden ebenfalls vermittelt. Dabei gaben sie an, sie nicht nur auf Pflegedienste hinzuweisen, sondern auch weitere lokale Hilfen für Angehörige zu kennen, die teilweise auf ehrenamtlicher Basis angeboten wurden. Die Praxisteams berichteten aber auch von fehlenden Angeboten oder negativen Erfahrungen. Fehlende Angebote bezogen sich laut Aussage der Praxisteams auf strukturelle Gegebenheiten, wie z.B. Brückenpflege und stationäre Einrichtungen der Palliativversorgung; negative Erfahrungen bezogen sich vorwiegend auf das Personal von Pflegediensten. Auch bürokratische Strukturen wurden von den Praxisteams als schwerfällig und zu wenig individuell beschrieben, auch wenn das Praxisteam sich besonders engagierte.

Tab. 3 Aufklärung, Unterstützung, Kontaktvermittlung.

\section{Code}

Hausärzte sensibilisieren die Angehörigen für den anstehenden Krankheitsprozess

Hinweis auf Hilfsmöglichkeiten für pflegende Angehörige

Fehlende lokale Angebote

Negative Erfahrungen mit Pflegediensten

Negative Erfahrungen mit bürokratischen Strukturen

\section{Beispielzitat}

„Die Situation kann man im Vorfeld ansprechen, wenn alles noch glatt läuft. Man sagt: Haben Sie mal überlegt, was passiert, wenn Sie morgen ausfallen, weil Sie sich das Bein gebrochen haben?" (HA10)

„Es gibt bei uns einen Gesprächskreis für pflegende Angehörige im Ort. Also einen ehrenamtlichen Kreis, wo die sich auch hinwenden können. “(HA9)

„Also wir haben kein Hospiz.“ (HA12)

„Da kommen aber immer ganz viele verschiedene Leute immer. Die glaub ich, bauen da nicht so arg die Beziehung auf wie ein privater Pflegedienst." (HA3)

„Ja, da haben wir dann festgestellt, dass es keine Pflegestufe gab. Also Krankenkasse sagt: Gibt's nichts. Medizinischer Dienst muss erstmal kommen. Ja, medizinischer Dienst hat gesagt, ich komm in 6 Wochen. Und dann hab ich da angerufen und gesagt: guter Mann, also in 6 Wochen braucht der Mensch das nicht mehr. Der braucht es jetzt und nicht in 6 Wochen. Der ist nicht gekommen. [...] Es war nicht möglich den MDK zu bewegen, jetzt in diesem aktuellen Fall wirklich mal eine Ausnahme zu machen und einfach mal morgen zu kommen und nicht in 6 Wochen. Es war nicht möglich. "(HA6)

Tab. 4 Verbesserungsbedarf (geäußerte Wünsche).

\section{Code}

Optimierung der Zusammenarbeit im professionellen Netzwerk kurzfristige Erreichbarkeit des Netzwerks Informationen über Angebote

Schulungen

Vergütung der Palliativversorgung

\section{Beispielzitat}

„Oder überhaupt eine Zusammenarbeit mit der Sozialstation, wir arbeiten eher gegeneinander, nicht zusammen. [...] ich auch für die, also ich nehme mich da nicht aus. Dass man einfach untereinander Verständnis hat." (MFA7)

„Also was ich mir wünschen würde, [...] dass man da wirklich akut so ein Netzwerk hat von Diensten und von Mitmenschen, die da einfach kompetent einspringen können und helfen können. [...] hauptsächlich den Angehörigen. " (HA2)

„[...] dass viele Angehörige auch gar nicht wissen, an wen sie sich wenden können. Dass da sicherlich auch noch Informationsdefizit ist. Was gibt es denn für Möglichkeiten? Wer hilft mir denn da? Also, dass diese Netzstrukturen, dass man die einfach mehr publik macht." (HA8)

„Also ich denk was hilfreich ist, wenn man dann Kärtchen oder [...] so paar Nummern hat, [...] allein schon dass das abgenommen wird, dass [der Patient] dann noch im Telefonbuch sucht. Natürlich kann er das. Logisch. [...] Oder hier das ist der Pflegestützpunkt. Rufen Sie doch mal die Frau Soundso an. Wenn die dann merken, die kennt man vielleicht. Das ist vertrauensbildend [...]. Und eine Hürde weniger." (HA9)

„Also, Hemmnisse finde ich schon manchmal die unzureichende Schulung der Sozialdienste im Gemeindepflegebereich, die sich mit der Palliativmedizin eigentlich nicht sehr gut auskennen, manchmal da ihre eigenen Ideen entwickeln. Aber nach Ausführen und ohne Rückfrage manchmal auch kontraproduktiv sind. [...] Da ist viel, viel Schulungsbedarf da. "(HA12) "und ich finde es die Palliativmedizin, ja dass das einfach ein teures Hobby ist. “(HA8)

„Wir würden das ja alles nicht bezahlt kriegen. Ich könnt unendlich mehr machen. Aber wir machen schon deutlich mehr. Ich sag jetzt für das Geld, was wir bekommen, machen wir bei solchen Patienten deutlich mehr als das, was wir bezahlt bekommen. [...] dass man dann noch zusätzlich die Familiengeschichte daueranalysiert, da noch eine Stunde Zeit nimmt, ja was können Sie da abrechnen? Gar nichts. Null. Fertig. [HA6: Ja] Ja.Und wenn man das ausbauen würde, das überleben Sie nicht. Das überleben Sie betriebswirtschaftlich nicht. Sie haben auch Verantwortung gegenüber Ihren Mitarbeitern. “(HA9) 
(3) Einbezug des sozialen Netzwerks der Angehörigen Neben den lokalen professionellen Angeboten führten die Praxisteams an, die Angehörigen wiederholt darauf hinzuweisen, ihr eigenes soziales Netzwerk zur Entlastung heranzuziehen. Sie argumentierten, dass damit Freiräume für die Angehörigen geschaffen werden könnten, die sie benötigten, um ihren eigenen Bedürfnissen nachzukommen.

„Familie oder Nachbarn oder was es halt so gibt, Freunde z.B., die man mit einbeziehen kann. Hier sind ja viele in irgend so einem Verein.“ (T1_HA13)

\section{(4) Verbesserungsbedarf (geäußerte Wünsche)}

Die Praxisteams nannten als Wunsch eine bessere Zusammenarbeit im professionellen Netzwerk ( $\bullet$ Tab. 4). Dabei appellierten sie sowohl an die Kollegialität der Ärzteschaft untereinander, warben aber auch um gegenseitiges Verständnis im Umgang mit Sozialstationen. Sie stellten fest, dass eine wichtige Eigenschaft eines entlastenden Netzwerkes eine kurzfristige Erreichbarkeit wäre. Das Netzwerk beschrieben sie als zusammengesetzt aus professionellen und nicht-professionellen Strukturen. Die Praxisteams erklärten, dass pflegende Angehörige bei Bedarf über entlastende Netzwerke und Angebote informiert werden sollen, vor allem über Selbsthilfegruppen und Schulungen für Angehörige. Die Praxisteams merkten dabei auch mögliche Vorteile für ihre eigene Arbeit an, z.B. eine Entlastung durch weniger Nachfragen vonseiten unsicherer Angehöriger. In diesem Zusammenhang erwähnten die Praxisteams die Notwendigkeit von ausreichenden Schulungen für alle Akteure im Netzwerk die Praxisteams sprachen hier vor allem Mitarbeiter von Sozialdiensten an.

Die Praxisteams (vor allem die Praxisinhaber) betonten die unzureichende Vergütung der Palliativversorgung, vor allem im Hinblick auf die Angehörigen, die zum Zeitpunkt der Fokusgruppen und Interviews nicht abrechnungsrelevant waren.

Die Praxisteams sahen trotzdem auch Optionen, die Praxisangebote für Angehörige zu verbessern. Zum einen sprachen sie die Möglichkeit an, die MFA stärker in die Betreuung der Angehörigen einzubinden, indem diese organisatorische Aufgaben übernehmen und auf Hilfsangebote verweisen könnten. Zum anderen wurde ein standardisiertes Vorgehen diskutiert, um den Umgang mit Angehörigen zu erleichtern. Ganz konkret wurde vorgeschlagen, für Angehörige und Patienten Adressen und Telefonnummern von Anlaufstellen schriftlich bereitzuhalten.

\section{Diskussion}

$\nabla$

Hausärztliche Praxisteams aus Hausärzten und Medizinischen Fachangestellten sind nach eigener Einschätzung in der Lage, überforderte pflegende Angehörige zu entlasten. Sie erkannten Bedürfnisse der Angehörigen, klärten sie über den anstehenden Krankheitsprozess auf, böten Unterstützung und vermittelten Kontakte zu weiteren Dienstleistern, mit denen die Zusammenarbeit aus ihrer Sicht weiter verbessert werden könnte.

Das Erkennen von Überforderung bei den Angehörigen geschieht nicht systematisch, sondern eher als Reaktion auf Überforderungsverhalten, das die Angehörigen den Praxisteams gegenüber zeigen. Dabei versuchen die Teams frühzeitig (vor der Überlastung), die Angehörigen auf kommende Herausforderungen der Pflegesituation hinzuweisen.

Bereits zu einem frühen Zeitpunkt im Krankheitsverlauf des Patienten klärten sie die Angehörigen über die zukünftige
Entwicklung auf, böten Unterstützung und vermittelten Kontakte zu lokalen Beratungs- und Betreuungsangeboten. Sie wiesen die Angehörigen auf deren soziales Netzwerk hin, damit sie sich Freiräume für ihre eigenen Bedürfnisse schaffen könnten. Damit greifen die Praxisteams Empfehlungen der DEGAM-Leitlinie auf [20] (siehe Box) und gehen auf den Wunsch der Angehörigen nach offener Kommunikation und Unterstützung ein [23].

\section{Ergebnisse mit Bezug zu * Empfehlungen der DEGAM-Leitlinie \\ - gezielte Nachfrage nach Belastungen* \\ - Beobachtungen (Veränderungen der Stimmungslage *, ver- ändertes Verhalten bei Praxisbesuchen*, Verhältnis zwi- schen Patienten und Angehörigen verschlechtert sich*,Überforderung mit Rollenvielfalt, steigende Kontaktfre- quenz, akute Belastungsreaktion*, Absprachen werden nicht mehr eingehalten) \\ - Angehörige berichten eigene Symptome* \\ - Sensibilisierung für den anstehenden Krankheitsprozess \\ - Psychosoziale Unterstützung * \\ - Hilfsmittel ${ }^{*}$, Koordination, Vermittlung von Beratungs- und Betreuungsangeboten* \\ - Einbezug des sozialen Netzwerks der Angehörigen*}

Den Praxisteams sind viele lokale und gesetzliche Unterstützungsmöglichkeiten bekannt. Nicht alle Entlastungsstrategien werden umgesetzt; teilweise können sie nicht umgesetzt werden, da aus ihrer Sicht dafür notwendige Strukturen fehlen oder noch nicht aufgebaut sind. So fehlen aus ihrer Perspektive nötige Hospizplätze. Die Unterstützung durch ein (professionelles) Netzwerk ist derzeit aus der Perspektive des Hausarztteams unzureichend. Die Unterstützung durch nichtmedizinische Angebote ist auch in Interviews mit Hausärzten einer anderen deutschen Studie gefordert worden [24]. Ist eine Vernetzung des Praxisteams mit anderen Dienstleistern vorhanden, entlastet dieses nicht nur die Angehörigen, sondern auch die Praxen selbst. Forderungen, die an das Praxisteam gestellt, und Wünsche, die an sie herangetragen werden, könnten dannan kompetente Akteure im Netzwerk weitergeleitet werden. So könnte eine stärkere Zusammenarbeit mit den Pflegekassen, die Hausärzte teilweise im Rahmen der Pflegeberatung heranziehen [25], und den lokalen Pflegestützpunkten die Entlastung der Angehörigen unterstützen.

Palliativversorgung findet in einem komplexen Versorgungssetting mit vielen Einflussvariablen statt. Dabei sind Hausarztpraxen nur ein Element unter unterschiedlichen Akteuren, wobei Patienten und Angehörige im Mittelpunkt stehen. In der vorliegenden Studie wurde der Fokus auf den Einfluss der Hausarztpraxen gelegt, da sie in vielen Fällen der wichtigste medizinische Ansprechpartner für zu Hause betreute Palliativpatienten sind. Nicht nur in Deutschland, sondern auch international fehlen Längsschnittstudien, die Ärzte, Palliativpatienten und Angehörige einbeziehen, um die Entwicklung von Interventionen zu unterstützen [26], die die Lebensqualität von Patienten und deren Angehörigen aufrechterhalten bzw. erhöhen und Einweisungen aufgrund der Überbelastung der Angehörigen verhindern oder aufschieben [27]. Eine Ausnahme ist die Implementierung des Gold Standards Framework in Großbritannien, in dem Angehörige explizit eingebunden sind [28]. Ihre Sichtweisen und Erfahrungen wurden bei der Entwicklung berücksichtigt, z.B. die 
Notwendigkeit eines professionellen Koordinators [29] und die Unterstützung durch district nurses [30]. Inwiefern solche Ansätze im stärker fragmentierten deutschen Gesundheitssystem übernommen oder gänzlich neu entwickelt werden müssen, ist Bestandteil zukünftiger Forschungsprojekte.

Unsere Ergebnisse zeigen, dass Hausärzte und MFA in der Identifikation belasteter pflegender Angehöriger und der Vermittlung von Unterstützungsmöglichkeiten eine Vielzahl von individualisierten Herangehensweisen aufweisen. Selbst besonders engagierte Praxisteams verfolgen eher einen reaktiven und keinen systematischen Ansatz. Die Ergebnisse der Fokusgruppen dienten als Grundlage für Folgeuntersuchungen im Rahmen des Projekts, um systematische Ansätze zu identifizieren und zu erproben [21].

Bisher erfassen die Praxisteams die (potentielle) Überforderung der pflegenden Angehörigen nicht systematisch. Ein Verweis in der Patientenakte, ob jemand pflegender Angehöriger bzw. welcher Angehörige des Patienten hauptsächlich für die Betreuung zuständig ist, könnte hier Abhilfe schaffen und einen ersten Hinweis darauf geben, auf wen hinsichtlich Überforderung durch eine Pflegesituation besonders zu achten ist. Pflegende Angehörige könnten beim Erstkontakt und bei Hausbesuchen proaktiv durch das Praxisteam identifiziert werden, aber auch durch aktives Nachfragen nach Unterstützern bei der pflegebedürftigen Person [31]. Die systematische Erfassung von Belastungen und Ressourcen pflegender Angehöriger und die Vernetzung mit anderen Dienstleistern und die Kenntnis ihrer Angebote erleichtern eine angemessene Intervention [32].

\section{Limitationen}

Die Ergebnisse dieser qualitativen Studie wurden aus forschungsökonomischen Gründen im Rahmen eines Pilotprojekts mit einer hoch selektierten Stichprobe gewonnen und lassen sich daher nicht unbedingt verallgemeinern. Die sehr engagierten Praxisteams bemühen sich eventuell stärker um eine (kollegiale) Vernetzung und sehen die (Mit-)Betreuung von pflegenden Angehörigen eher als ihre Aufgabe an als Praxisteams, die keine Qualifikation oder ein besonderes Augenmerk auf die Palliativversorgung haben. Da sich die angesprochenen Themen in den Fokusgruppen größtenteils überschneiden, kann für diese Stichprobe von einer Sättigung ausgegangen werden.

Durch die den Fokusgruppen vorangegangene Informationsveranstaltung wurden die Teilnehmer bereits für das Thema sensibilisiert. Bei den Informationsveranstaltungen ist aber darauf geachtet worden, bereits bestehende Unterstützungsansätze (z.B. die DEGAM-Leitlinie Pflegende Angehörige) zu benennen, ohne detaillierter darauf einzugehen. In den Fokusgruppen ist wiederum die Konzentration auf persönliche Erfahrungen betont worden. Die Leitlinie ist in den Gesprächen selbst weder von den Forscher/innen noch von den Teilnehmer/innen explizit angesprochen worden.

Im Gesprächsverhalten zeigten sich Unterschiede zwischen Hausärzten und Medizinischen Fachangestellten. Die Hausärzte dominierten im Gespräch. Das lässt vermuten, dass der soziale Status den Gesprächsverlauf dahingehend beeinflusste, dass die Redeanteile unterschiedlich gewichtet waren.

Andererseits konnten wir hier auch nur die Sicht der Praxisteams darstellen - inwieweit ihre Wahrnehmung, z.B. von Hilfsangeboten, faktischen Gegebenheiten entspricht, lässt sich in diesem Rahmen nicht überprüfen. Genauso bräuchte es eine
Triangulation mit Patienten- und Angehörigenbefragungen, um die Reichweite der Praxisangebote zu eruieren.

\section{Schlussfolgerung \\ $\nabla$}

Die Belastung und die Ressourcen der pflegenden Angehörigen sind individuell verschieden und bedürfen einer individuellen Erfassung und eines frühzeitigen, maßgeschneiderten Ansatzes zur Entlastung. Die Praxisteams dieser Studie bieten nach eigener Aussage Angehörigen bereits viele Unterstützungsmöglichkeiten an und verweisen sie an weitere Anlaufstellen. Die Kenntnis der lokalen Gegebenheiten und eine gute Vernetzung des Praxisteams mit anderen professionellen Anbietern können zu einer verbesserten Versorgung beitragen. Diese Unterstützungsangebote bedürfen einer Systematisierung, sodass Beratungsgespräche strukturiert und trotzdem auf die individuellen Bedürfnisse der Betroffenen zugeschnitten durchgeführt werden können. Für einige Angehörige werden Hinweise und schriftliche Informationen ausreichen, andere werden mehr Unterstützung benötigen, für die psychosoziale Kompetenzen in Fort- und Weiterbildung vermittelt werden sollten. Der Aufwand für eine solche individuelle Betreuung von Angehörigen sollte stärker in der Vergütung der Praxisteams berücksichtigt werden.

Interessenkonflikt: Die Autoren geben an, dass kein Interessenkonflikt besteht.

\section{Literatur}

1 Gomes B, Higginson IJ. Factors influencing death at home in terminally ill patients with cancer: systematic review. BMJ 2006; 332: 515-521

2 Schneider N, Mitchell GK, Murray SA. Palliative care in urgent need of recognition and development in general practice: the example of Germany. BMC family practice 2010; 11: 66

3 Murray MA, Fiset V, Young $S$ et al. Where the dying live: a systematic review of determinants of place of end-of-life cancer care. Oncol Nurs Forum 2009; 36: 69-77

4 Döhner H, Kofahl C, Lüdecke D et al. The National Survey Report for Germany. In, EUROFAMCARE Services for Supporting Family Carers of Older Dependent People in Europe: Characteristics, Coverage and Usage 2007

5 Bleidorn J, Klindworth $K$, Krenz $S$ et al. Versorgung am Lebensende: Bedürfnis nach „sprechender Medizin“. Dtsch Arztebl International 2011; 108: 1225-1226

6 Docherty A, Owens A, Asadi-Lari $M$ et al. Knowledge and information needs of informal caregivers in palliative care: a qualitative systematic review. Palliat Med 2008; 22: 153-171

7 Lavela SL, Ather N. Psychological health in older adult spousal caregivers of older adults. Chronic Illn 2010; 6: 67-80

8 Gröning $K$. Therapeutisierung der familialen Altenfürsorge? Formulierung eines Unbehagens? Sozialer Fortschritt 2005; 3: 69-76

9 Schlarmann JG, Metzing S, Schoppmann S et al. Germany's First Young Carers Project's Impact on the Children: Relieving the Entire Family. A Qualitative Evaluation. The open nursing journal 2011; 5: 86-94

10 Schnepp W, Duijnstee M, Grypdonck M. Migrationsspezifische Transitionen und Angehörigenpflege. Pflege 2005; 18: 305-312

11 Zank S, Schacke C. Projekt Längsschnittstudie zur Belastung pflegender Angehöriger von demenziell Erkrankten (LEANDER). Abschlussbericht Phase 2: Längsschnittergebnisse der LEANDER Studie In: 2007

12 Menn P, Holle R, Kunz S et al. Dementia care in the general practice setting: a cluster randomized trial on the effectiveness and cost impact of three management strategies. Value in health: the journal of the International Society for Pharmacoeconomics and Outcomes Research 2012; 15: 851-859

13 Anonym. Ermutigende Ergebnisse - Pflegende Angehörige von Demenzkranken können entlastet werden. Z Palliativmed 2010; 11: 10

14 Sörensen S, Pinquart M, Duberstein P. How effective are interventions with caregivers? An updated meta-analysis. Gerontologist 2002; 42: 7

15 Clemens KE, Klaschik E. Entwicklungsstand im Jahr 2007. Anasthesiol Intensivmed Notfallmed Schmerzther 2007; 42: 280-285 
16 Kern $M$, Wessel H, Ostgathe E. Ambulante Palliativbetreuung - Einflussfaktoren auf eine stationäre Einweisung am Lebensende. Z Palliativmed 2007; 8: 7

17 Howat A, Veitch C, Cairns W. A retrospective review of place of death of palliative care patients in regional north Queensland. Palliat Med 2007; $21: 41-47$

18 Jordhoy MS, Fayers $P$, Saltnes $T$ et al. A palliative-care intervention and death at home: a cluster randomised trial. Lancet 2000; 356: 888-893

19 Tiernan E, O'Connor M, O'Siorain L et al. A prospective study of preferred versus actual place of death among patients referred to a palliative care home-care service. Ir Med J 2002; 95: 232-235

20 Anonym. Pflegende Angehörige. Leitlinie Langfassung. Düsseldorf: omikron publishing; 2005

21 Hermann $K$, Boelter $R$, Engeser $P$ et al. PalliPA: How can general practices support caregivers of patients at their end of life in a home-care setting? A study protocol. BMC Res Notes 2012; 5: 233

22 Mayring P. Qualitative Inhaltsanalyse. In: Flick U, Ev Kardoff, Steinke I, (Hrsg.). Qualitative Forschung Ein Handbuch. 5. AuflReinbek: Rowohlt Taschenbuch; 2007

23 Bleidorn J, Pahlow H, Klindtworth K et al. Versorgung von Menschen am Lebensende. Erfahrungen und Erwartungen von hinterbliebenen Angehörigen. Dtsch Med Wochenschr 2012; 137: 1343-1348

24 Götze H, Perner A, Gansera L et al. „Da kann man nicht auf die Uhr gucken“ - Interviews mit Hausärzten zur ambulanten Palliativversorgung von Tumorpatienten. Gesundheitswesen (Bundesverband der Arzte des Offentlichen Gesundheitsdienstes (Germany)) 2013; 75: 351-355
25 GKV Spitzenverband der Pflegekassen. Evaluation der Pflegeberatung nach § 7a Abs. 7 Satz 1 SGB XI. Evaluationsbericht des AGP Institut für angewandte Sozialforschung, Hans Weinberger-Akademie, TNS Infratest. Im Internet: https://www.gkv-spitzenverband.de/media/ dokumente/pflegeversicherung/beratung_und_betreuung/pflege beratung/Evaluation_der_Pflegeberatung_Dezember_2011__7a_Abs_ SGB_XI.pdf Zugriff: 17.07.2015;

26 Grande G, Stajduhar K, Aoun S et al. Supporting lay carers in end of life care: current gaps and future priorities. Palliat Med 2009; 23: 339-344

27 Jack B, O'Brien $M$. Dying at home: community nurses' views on the impact of informal carers on cancer patients' place of death. Eur J Cancer Care (Engl) 2010; 19: 636-642

28 Thomas K. Caring for the dying at home. Oxford: Radcliffe: Publishing; 2003

29 Boyd KJ, Worth A, Kendall $M$ et al. Making sure services deliver for people with advanced heart failure: a longitudinal qualitative study of patients, family carers, and health professionals. Palliat Med 2009; 23: 767-776

30 Griffiths J, Ewing G, Rogers M. Early support visits by district nurses to cancer patients at home: a multi-perspective qualitative study. Palliat Med 2013; 27: 349-357

31 Höppner C, Schneemilch M, Lichte T. Pflegende Angehörige und ihre Belastungen in Hausarztpraxen identifizieren - Hindernisse und Empfehlungen. Z Allg Med 2015; 91: 310-313

32 Monteverde M, Fringer A. Zu Hause sterben: pflegende Angehörige befähigen. palliat ch 2013; 2: 34-37 